\title{
A Multi-Environment Thermal Control System With Freeze-Tolerant Radiator
}

\author{
Weibo Chen, David Fogg, Nick Mancini \\ Creare Inc., Hanover, New Hampshire, 03755 \\ John Steele, Gregory Quinn \\ United Technologies Corporation Aerospace Systems, Windsor Locks, Connecticut, 06096 \\ Grant Bue, Sean Lillibridge \\ NASA Lyndon B. Johnson Space Center, Houston, Texas, 77062
}

\begin{abstract}
Future space exploration missions require advanced thermal control systems (TCS) to dissipate heat from spacecraft, rovers, or habitats operating in environments that can vary from extremely hot to extremely cold. A lightweight, reliable TCS is being developed to effectively control cabin and equipment temperatures under widely varying heat loads and ambient temperatures. The system uses freeze-tolerant radiators, which eliminate the need for a secondary circulation loop or heat pipe systems. Each radiator has a self-regulating variable thermal conductance to its ambient environment. The TCS uses a nontoxic, waterbased working fluid that is compatible with existing lightweight aluminum heat exchangers. The TCS is lightweight, compact, and requires very little pumping power. The critical characteristics of the core enabling technologies were demonstrated. Functional testing with condenser tubes demonstrated the key operating characteristics required for a reliable, freeze-tolerant TCS, namely (1) self-regulating thermal conductance with short transient responses to varying thermal loads, (2) repeatable performance through freeze-thaw cycles, and (3) fast start-up from a fully frozen state. Preliminary coolant tests demonstrated that the corrosion inhibitor in the water-based coolant can reduce the corrosion rate on aluminum by an order of magnitude. Performance comparison with state-of-the-art designs shows significant mass and power saving benefits of this technology.
\end{abstract}

\section{Introduction}

Future spacecraft, rovers, and habitats will require high-performance thermal control systems that can dissipate a wide range of heat loads in harsh thermal environments. Environmental temperatures can vary by more than $200 \mathrm{~K}$ during each environmental cycle (Stephan 2011) and heat loads can change by an order of magnitude when the power supply mainly comes from solar panels. However, cabin temperatures must be controlled within a narrow range to ensure the comfort of crew members and to reduce thermal stresses in electronic components and other equipment. Therefore, a future TCS must be able to vary the thermal conductance between the cabin and the ambient over a wide range. Furthermore, when the cabin heat load is low and ambient is very cold, temperatures in radiators can fall below the freezing point of the working fluid. Fluid freezing poses many hazards. One such hazard is potential rupture of the radiator tubes due to the expansion of the working fluid during local freezing or thawing processes. Another problem is the very slow thawing process during which the TCS will not be able to achieve its full heat dissipation capacity.

Several approaches have been studied to achieve variable thermal conductance and freeze-tolerance. For example, a two-loop configuration was studied for NASA Altair Lunar Lander (Stephan 2011). This approach uses a secondary circulation loop with working fluid that has a freezing temperature well below the minimum environment temperature that the radiator would experience. This requires an interface heat exchanger between the loops and a separate circulation pump for the secondary loop. Furthermore, working fluids with low freezing temperatures typically have poor thermal and fluid properties, requiring larger heat exchangers or higher pumping power. A variation of this approach uses loop heat pipes to eliminate the circulation pump; however, this adds complexity during start-up and mass to the system. Other approaches to prevent freezing include (1) using survival heaters to prevent working fluid from freezing, (2) actively controlling radiator conductance by varing the radiator exposed 
surface areas or surface effective emissivity (Bannon et al. 2010), (3) removing working fluid from some of the radiator tubes to reduce active areas and therefore the heat dissipation capacity (Ganapathi et al. 2009), and (4) allowing working fluid to freeze on the internal surfaces of radiator tubes while maintaining a small liquid flow through the center of the tubes (Nabity et al. 2008). However, all these methods incur some performance penalties in terms of mass, power, reliability, and recovery time, or pose technical challenges due to their low TRL. For these reasons, there is a strong need for an alternative lightweight, reliable TCS that self regulates its thermal conductance and can tolerate freezing of its working fluid.

\section{TCS With Freeze-Tolerant and Varaiable-Conductance Radiator}

A single-loop TCS with freeze-tolerant radiator that has variable thermal conductance is being developed at Creare. As shown in Figure 1, the TCS uses a single-phase circulation loop inside the cabin for heat acquisition and transfer, and a low-pressure, two-phase loop outside the cabin for heat rejection. Single-phase circulating water in the hot portion of the circulation loop is cooled in a membrane evaporator by evaporating a very small fraction of the circulating water. The resulting water vapor condenses in the freeze-tolerant condenser, which has a selfregulating thermal conductance to control the heat rejection rate. The condenser has unique design features that allow water vapor to flow into the condenser and condensate to flow out of the condenser even when a large section of the condenser tube is frozen, thus enabling the radiator to continue to work effectively. An ejector pump then draws the condensate from the external condenser back into the main loop. Each radiator passively self-regulates its thermal conductance to the ambient environment, giving them heat rejection turndown ratios higher than 20 . The pumped coolant loop uses a benign working fluid that consists mainly of water with a very low concentration of corrosion inhibitors and biocides, which ensures the working fluid is compatible with existing aluminum heat exchangers, crew health, and vehicle systems.

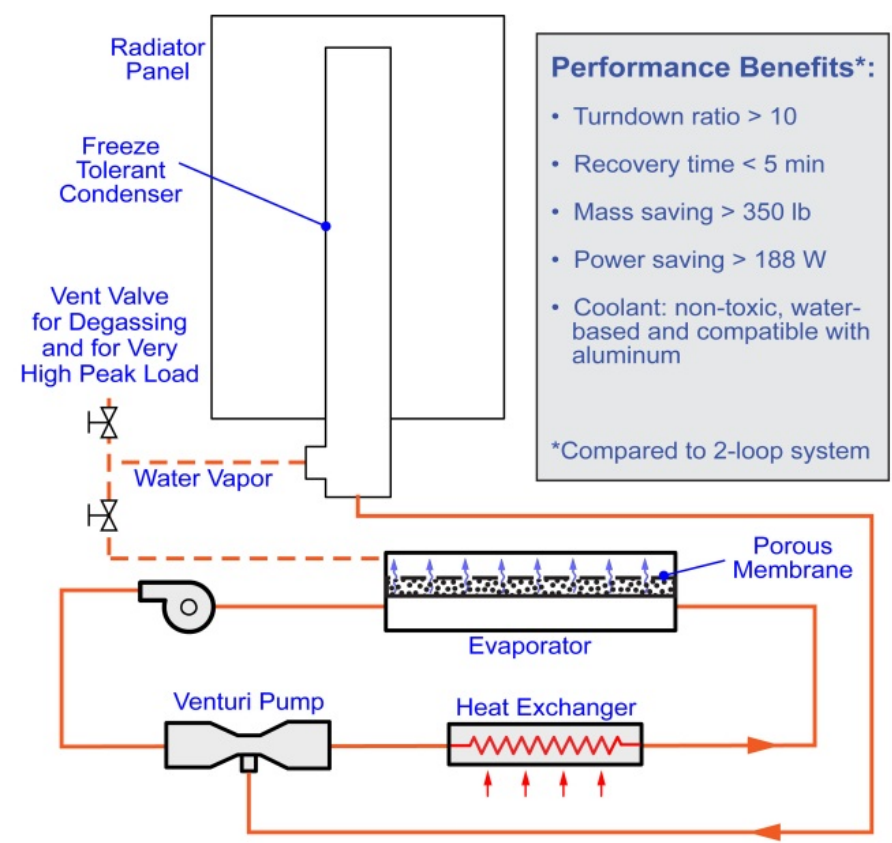

Figure 1. Layout of a Single-Loop TCS With Freeze-Tolerant Condensers/Radiators Having a Variable Thermal Conductance

The TCS also can provide a very high cooling power if needed by directly venting water vapor to the external environment. The vent valve in the TCS also allows venting of noncondensable gas in the condensers during scheduled maintenance.

The TCS combines components that are being developed at Creare, and technologies that have already been developed at NASA JSC and United Technologies Corporation Aerospace Systems (UTAS) to create a thermal control system that will meet the challenges of temperature control on future manned spacecraft. The three key elements in the TCS and their features are discussed below. 
Subcooled, Single-Phase Loop Inside Cabin. The single-phase loop inside the cabin is pressurized to a level high enough to provide sufficient subcooling to prevent two-phase flow in this loop. This allows the use of simple singlephase heat exchangers to absorb heat. Using a single-phase loop also eliminates potential flow instability associated with two-phase flows and thus simplifies system design, operation, control, and maintenance. This is especially true in a system that has multiple cold plates with different flow resistances and heat loads. The subcooling also prevents cavitation in circulation pumps, simplifying pump design and enhancing pump reliability. The pumps control the loop circulation flow rate to maintain the cabin temperature. These design features lead to a compact, lightweight TCS with a large heat transfer capacity.

Direct Evaporative Chiller. Heat rejection from the single-phase loop is accomplished by direct evaporative cooling. A small fraction of the circulation water evaporates to cool the bulk circulating flow. The resulting water vapor flows to the parallel radiation-cooled condensers outside the cabin. The condensate is drawn back to the loop inside the cabin with a passive, cavitation-tolerant ejector pump. For a fixed evaporation pressure, the heat rejection rate in the evaporative chiller can be controlled by the circulation water flow rate. Due to the high heat transfer coefficient associated with the evaporation process, the evaporative chiller is far more compact than a single-phase, liquid-to-liquid heat exchanger in a conventional system using a secondary loop. Furthermore, for a given size radiator panel, the maximum heat rejection capacity of a two-phase flow is appreciably higher than that of a single-phase flow. This is because the temperature of a two-phase flow does not decrease as it flows across the condenser, while a single-phase flow always does. Compared to a system using heat pipes (including loop heat pipes) in its external secondary loop, our system eliminates the need for interface heat exchangers, heat pipe accumulators, and bypass control needed in extremely cold environments. Current heat pipes also have the shortcoming of limited heat transport capacity and operating stability issues associated with multiple condensers.

The configuration of the internal portion of our TCS is similar to the liquid cooling loop in future spacesuits incorporating the Spacesuit Water Membrane Evaporator (SWME) currently under study by NASA for evaporative cooling (Bue and Makinen 2011). Our TCS eliminates water venting by incorporating radiation cooled condensers to condense water vapor and draw the condensate back inside the cabin. Minimizing consumables is critical for future long-duration exploration missions.

Freeze-Tolerant Condensers/Radiators. The radiation cooled condenser is freeze-tolerant and has design features that self-regulate its thermal conductance to the ambient environment. The variable conductance allows the TCS to maintain a stable evaporation pressure for the evaporative chiller, and thus a stable cabin temperature even when the variations in cabin heat loads and ambient temperature are large. The freeze-tolerant condensers also enable water as working fluid. This eliminates the need for other working fluids with very low freezing temperatures that have much poorer thermophysical properties relative to water.

\section{Condenser/Radiator Design Optimization}

Condenser Design Features. The radiation-cooled condensers are designed to tolerate freezing of the working fluid and yet continue to reject heat. Compared with other radiator designs, the key difference in this condenser/radiator is the continuous connection between the vapor channel and the condensate return channel.

The condenser has three distinct operating regions. Close to the vapor inlet is the active region operating at the inlet vapor temperature; at the distal end is the inactive region at the effective sink temperature; and in between lies a short transition zone. The length of the active region is proportional to the condensation rate. It increases with TCS input power which leads to more vapor. The length of the active region also increases when the effective sink temperature is increased due to the lower local condensation heat flux. When the active regime extends to the distal end of the condenser tube, the condenser heat rejection capacity reaches its maximum value. This ability of the condenser to regulate the length of its own active region enables it to achieve a variable thermal conductance to the ambient environment.

With a relatively uniform heat sink temperature over the entire radiator panel, the far end of the condenser tube will always be at the lowest temperature of the entire tube. Therefore, water will tend to start to freeze at that end. The frozen section will gradually extend toward the vapor inlet as the heat load decreases or the ambient temperature decreases. This behavior will prevent water from being trapped between two iced sections. This feature prevents excessive stresses in the condenser tube associated with fluid expansion during freeze-thaw cycles.

Condenser Design Analysis. To maintain a comfortable cabin environment, the evaporative chiller needs to operate at a saturation temperature below $20^{\circ} \mathrm{C}$. The corresponding saturation vapor pressure is only 17.5 torr. Therefore, pressure drop within the vapor lines connecting the evaporative chiller to condensers must be limited to no more than 2 torr. This low-pressure drop requirement, coupled with the low density of water vapor, necessitates a condenser tube with a relatively large diameter. Other design constraints for the condenser/radiator, including the 
minimum tube wall thickness for the condenser, the minimum radiator panel thickness, and the minimum radiator panel length, are imposed in the radiator design optimization process.

Given these constraints, the design parameters for the panel are optimized for minimum mass for a given heat rejection rate. The optimal design is a $1 \mathrm{~m}$ long panel with a condenser tube pitch of $0.150 \mathrm{~m}$ capable of rejecting $38.4 \mathrm{~W}$ at full power with a heat sink temperature of $200 \mathrm{~K}$.

The transient thermal response of the radiator is calculated with a 2-D numerical model. Based on the panel dimensions, radiator heat load profile, saturation temperature, sink temperature, and panel initial temperatures, the analysis code predicts the time-varying radiator temperature profile, pressure drop, and the length of two-phase zone in the condenser.

Figure 2 shows the predicted thermal response of the radiator to a power ramp from $5 \mathrm{~W}$ to $35 \mathrm{~W}$. Before $\mathrm{t}=$ $5 \mathrm{~min}$, the radiator operates at a steady state with the two-phase zone extending only about $5 \mathrm{~cm}$ into the condenser and $90 \%$ of condenser length is at temperatures well below the freezing point. When the heat load is instantaneously raised to $35 \mathrm{~W}$, the two-phase region quickly advances and melts the ice downstream. Within less than 10 minutes, the two-phase zone expands to a length of about $80 \%$ of the condenser tube. This analysis confirms that the radiator can recover very quickly from a nearly fully frozen state. Figure 2 also shows the effect of latent heat of fusion in the freezing process $(t=120 \mathrm{~min})$. The pressure drop during the entire process remains less than $10 \%$ of the saturation pressure.

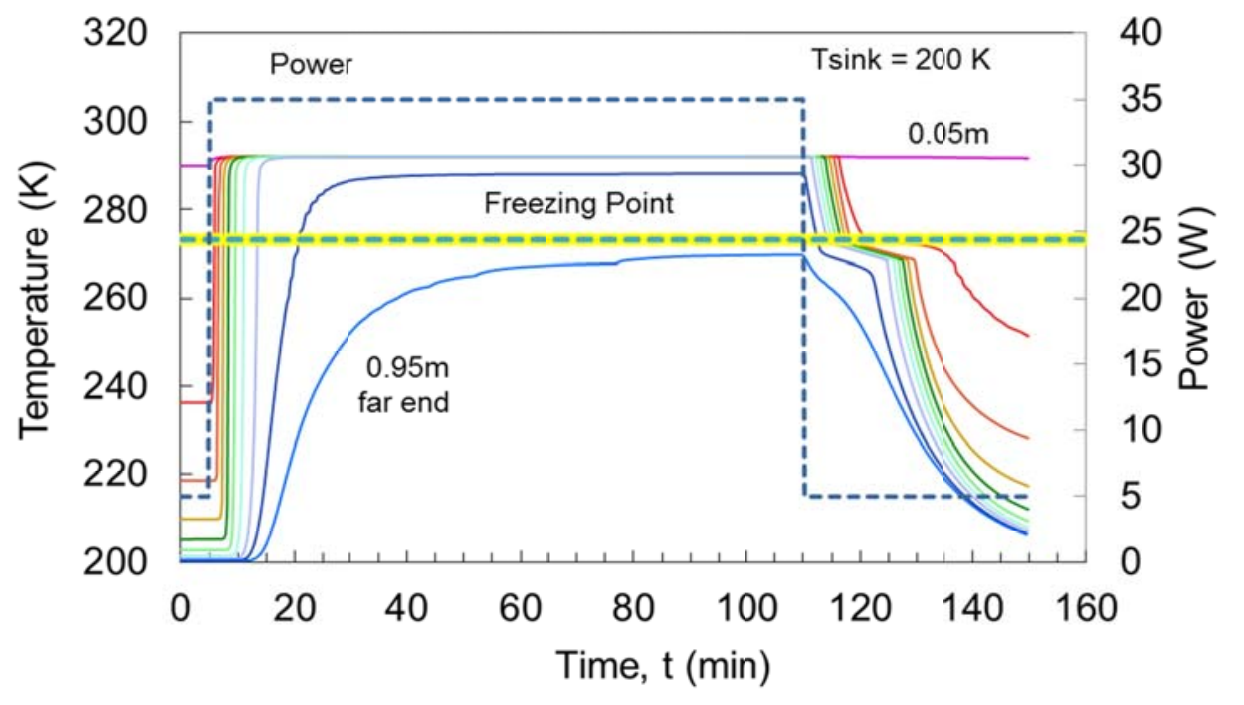

Figure 2. Predicted Response of Freeze-Tolerant Radiator to a Power Ramp. Solid lines represent temperatures at locations that are $10 \mathrm{~cm}$ apart. The first and last locations are $0.05 \mathrm{~m}$ and $0.95 \mathrm{~m}$ away from the vapor inlet.

Figure 3 shows the radiator responses to an input power step-down. As the power decreases, the length of the two-phase zone decreases approximately proportionally. The pressure drop across the radiator also decreases with power, as expected. At the input power of $20 \mathrm{~W}$, which is about $50 \%$ of the radiator maximum capacity, the length of the frozen zone is about $40 \%$ of the condenser length. 


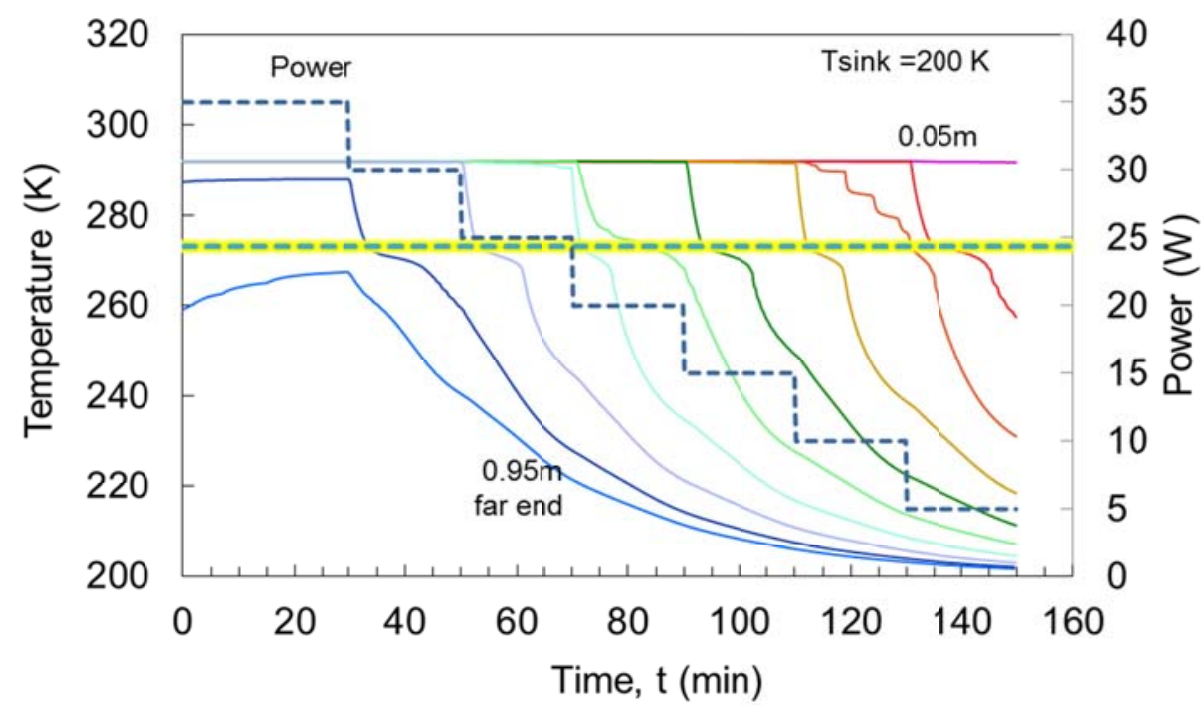

Figure 3. Predicted Response of Freeze-Tolerant Radiator to Power Step-Down. Solid lines represent temperatures at locations that are $10 \mathrm{~cm}$ apart. The first and last locations are $0.05 \mathrm{~m}$ and 0.95 away from the vapor inlet.

Several factors affect the transition time from one operating load to another with ice in the condenser. Axial conduction via the water, radiator panel, and condenser primarily dictates the melting rate. Recovery time is also affected by the tube cross-sectional area. Ideally, the cross-sectional area would be large enough to minimize the pressure drop through the active zone; however, as the tube diameter increases, the time to fill, freeze, and melt increases in proportion to volume stored. Fortunately, the latent heat of fusion provides ample thermal capacity to absorb the evaporator heat load that increases during the thaw process. Even though the time to thaw a large tube increases with diameter, the condenser can rely on the fusion heat to absorb its full heat load before the condenser is completely thawed, as shown in Figure 2 and the preliminary test results discussed later in this paper.

Mechanical Design. A preliminary mechanical design of the radiator demonstrator was developed, as shown in Figure 4. Each panel consists of a collection of parallel condenser tubes. Based on this design, the predicted mass of a $6 \mathrm{~kW}$ radiator system is approximately $50.5 \mathrm{lb}(23 \mathrm{~kg})$ and the panel area is $250 \mathrm{ft}^{2}\left(23 \mathrm{~m}^{2}\right)$.

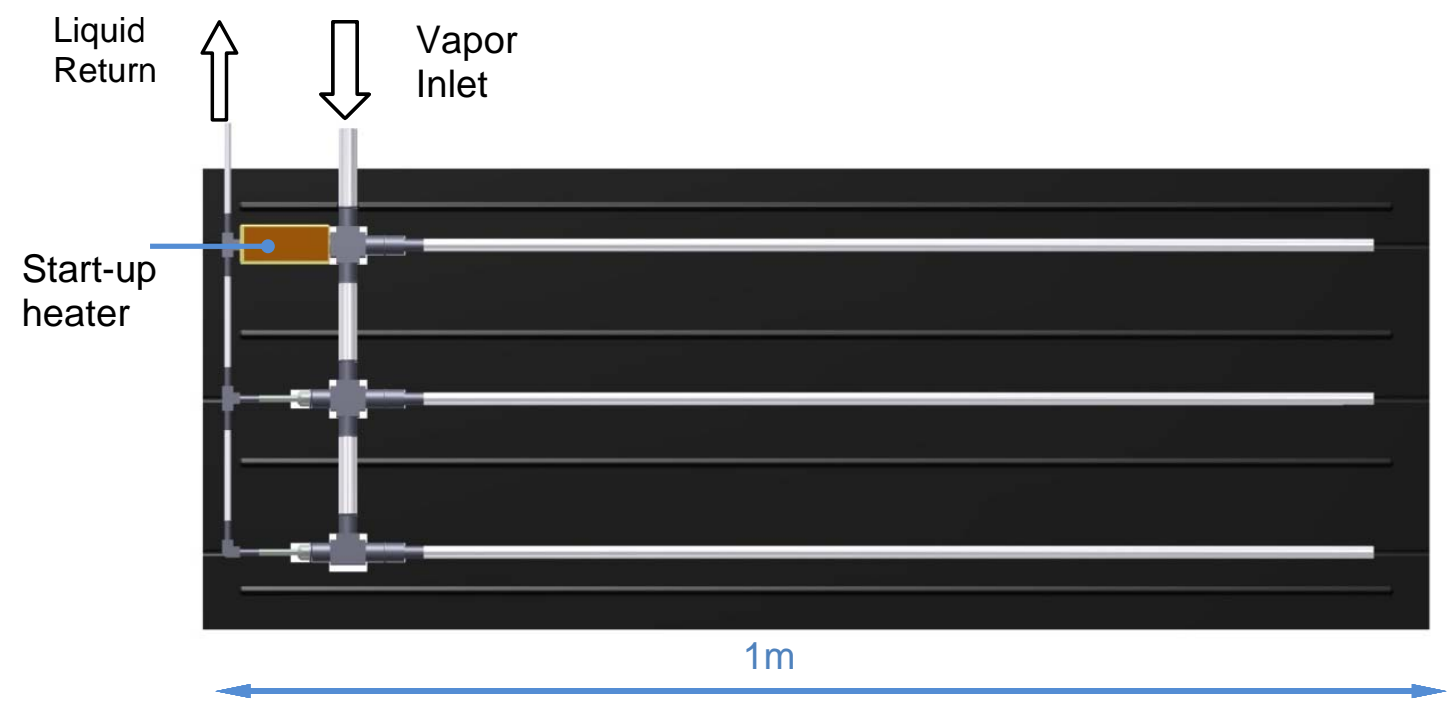

Figure 4. Preliminary Layout Design for Freeze-Tolerant Condenser/Radiator 


\section{Ejector Pump Design}

The ejector pump is a critical system component responsible for returning the low-pressure condensate in the radiator panel back to the high-pressure cabin loop. To raise the pressure of the condensate back to the cabin loop pressure, our approach is to use a single-stage ejector that uses a small flow of high-pressure water from the main circulation loop to raise the condensate pressure to an intermediate pressure, e.g., 10 psia, and then employ a small booster pump to complete the process. The intermediate pressure only needs to be high enough to avoid cavitation in the booster pump. For a $6 \mathrm{~kW}$ cooling system, the primary flow rate is only about $4 \mathrm{~g} / \mathrm{s}$, or $32 \mathrm{lb} / \mathrm{hr}$. This is only about $10 \%$ of the main pump flow rate. The ideal power input for the booster pump is only about $1.5 \mathrm{~W}$.

We completed scoping analyses to develop a preliminary ejector pump design that will recirculate radiator condensate with $5^{\circ} \mathrm{C}$ of subcooling using a primary motive stream at 45 psia. To prevent cavitation in the suction flow, which would increase suction flow volumetric flow rate and thus reduce the ejector entrainment ratio, we limit the maximum acceleration pressure drop from the suction inlet to the mixing chamber inlet to less than $0.05 \mathrm{psi}$. As a result, the suction flow area must be relatively large.

A preliminary ejector design, with dimensions determined from conservation laws and empirical diffuser performance maps, was analyzed with a CFD model for incompressible fluid. Figure 5 shows the ejector pump model. The nozzle diameter is only about $0.5 \mathrm{~mm}$.

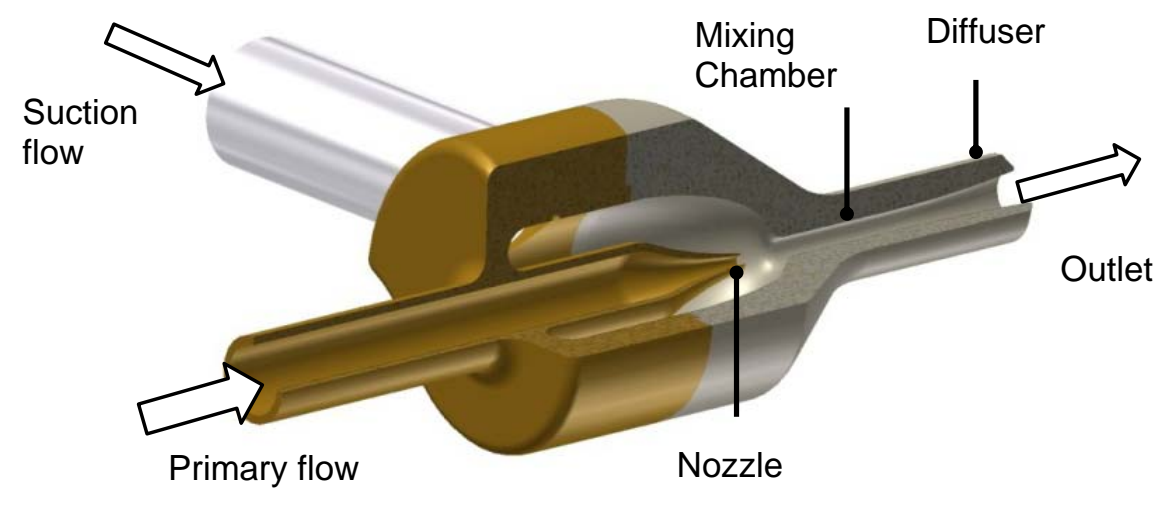

Figure 5. CAD Model of a Preliminary TCS Ejector Pump

The computational domain is axisymmetric and thus only a 20-degree section of the entire ejector was modeled. Mesh refinement near the walls and mixing regions resolves boundary layer growth (particularly in the diffuser), as well as the shearing region where the high-speed nozzle exit flow and the entrained suction flow meet. Pressure boundary conditions are imposed at the inlet (45 psia), suction inlet (0.26 psia), and diffuser outlet (11 psia), and the resulting mass flow rates were determined from the CFD results.

The resulting primary flow rate is $4.03 \mathrm{~g} / \mathrm{s}$ and the suction flow rate is $2.24 \mathrm{~g} / \mathrm{s}$, corresponding to an entrainment ratio of 0.56 with an ideal exit pumping power of $1.47 \mathrm{~W}$. Table 1 provides a summary of all key ejector performance data predicted by the CFD analysis.

Figure 6 shows the pressure contours within the ejector. The ejector design includes a larger suction throat which reduces the acceleration pressure drop and thus cavitation potential. The minimum pressure in this region is about $1600 \mathrm{~Pa}$ which corresponds to a saturation temperature of $14^{\circ} \mathrm{C}$. Short regions of sub-saturation pressures are allowable as the cavitation bubbles have fast, but finite growth rates. If the fluid transit period across the low-pressure region is sufficiently small, minute vapor bubbles might not have adequate time to grow before entering higher pressure regions where they will condense.

The CFD results were also used to iteratively determine the optimum length of the mixing section. The mixing section must be long enough to allow the suction flow and primary flow to reach the same speed and pressure. An excessively long mixing chamber, however, can cause large flow losses due to wall friction. We iteratively adjust the chamber length to maximize the ejector performance (i.e., maximize suction flow rate) for the given pressure boundaries. 


\begin{tabular}{|l|c|}
\hline \multicolumn{2}{|l|}{ Table 1. Ejector Pump CFD Predictions } \\
\hline \hline Overall Mechanical Efficiency & $18.2 \%$ \\
\hline Primary Mass Flow rate (g/s) & 4.03 \\
\hline Suction Mass Flow rate (g/s) & 2.24 \\
\hline Entrainment Ratio (-) & 0.56 \\
\hline Primary Flow Inlet Pressure (psia) & 44.9 \\
\hline Suction Pressure (psia) & 0.26 \\
\hline Outlet Pressure (psia) & 11.2 \\
\hline Minimum Suction Pressure (psia) & 0.232 \\
[Corresponding Tsat ( ${ }^{\circ}$ C)] & {$[14]$} \\
\hline Nozzle Bulk Exit Velocity (m/s) & 23.6 \\
\hline Nozzle Efficiency (-) & $90.4 \%$ \\
\hline Bulk Velocity Entering Diffuser (m/s) & 8.14 \\
\hline Diffuser Inlet Pressure (psia) & 8.0 \\
\hline
\end{tabular}

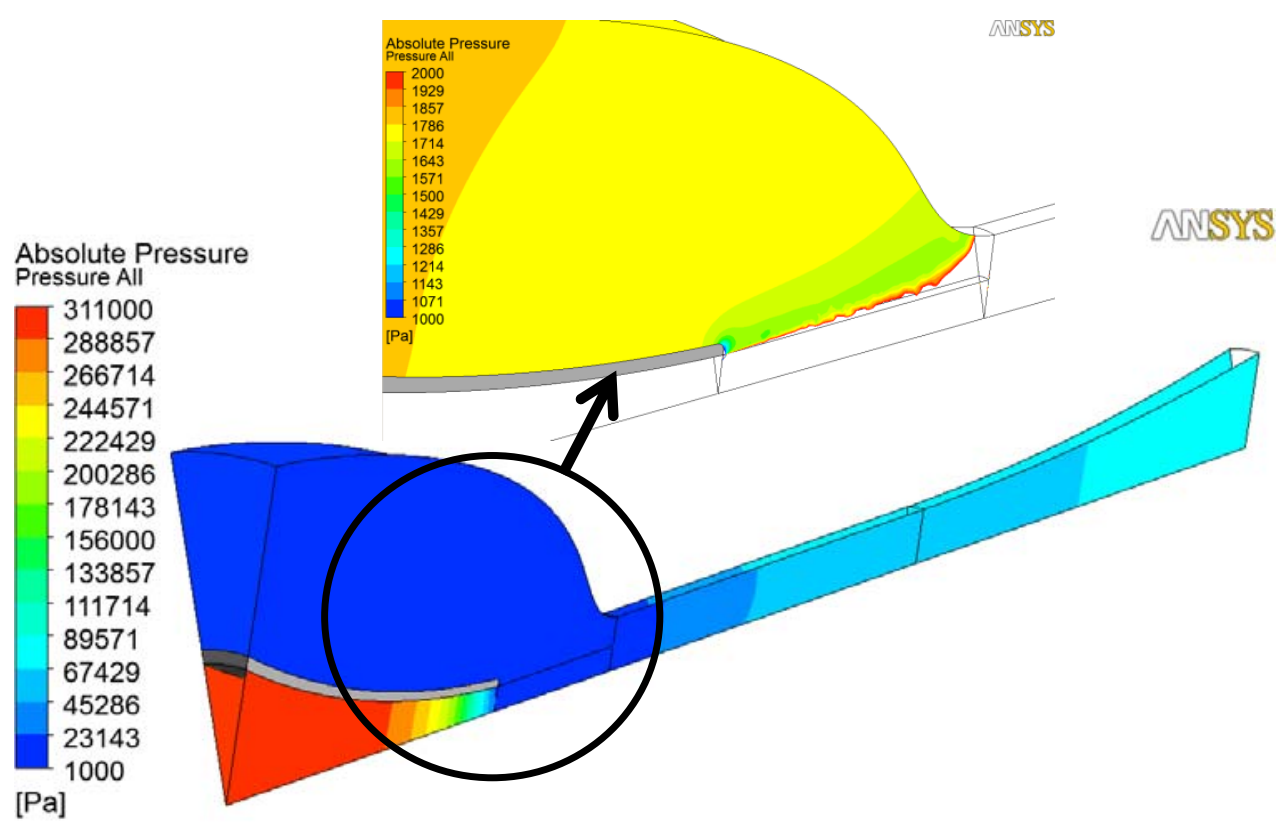

Figure 6. Ejector Pump Pressure Contours. Pressure in the region just upstream of the nozzle jet in the suction flow is approximately $1600 \mathrm{~Pa}$.

\section{Proof-of-Concept Freeze-Tolerant Demonstration}

Freeze-Tolerant Condenser. The proof-of-concept freeze-tolerant condenser prototype is a tube-in-shell style design (Figure 7). The concept demonstrator design incorporates the prototypical flow and heat transfer characteristics of the optimized radiator panel design. The demonstrator is convectively cooled rather than radiatively to simplify the test setup. However, the condenser shell is sized to approximate the axial conduction of an aluminum radiator panel. 


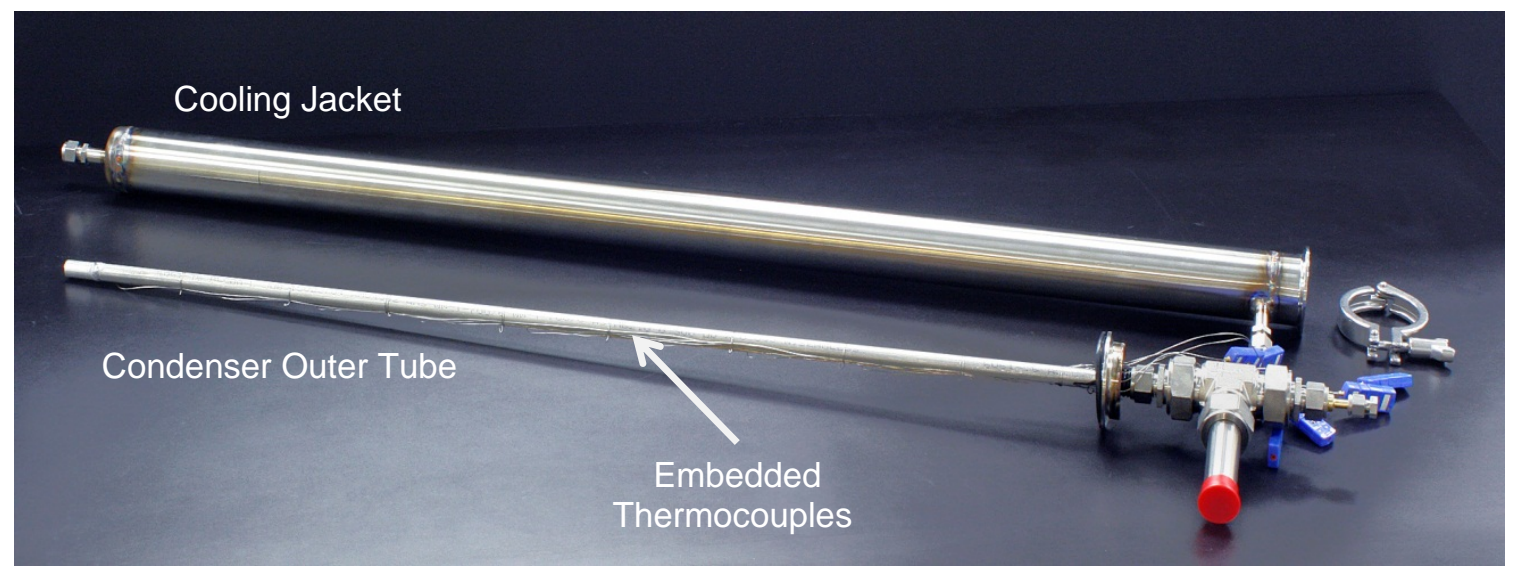

Figure 7. Phase I Prototype Condenser Design

Prototypical Condenser Test Facility. Figure 8 provides a schematic of the test facility to demonstrate the key features of the freeze-tolerant condenser. The major components consist of a low-pressure evaporator, a prototypical condenser tube, and a convectively cooled radiator load simulator.

The evaporator consists of a stainless steel tube, an internal circulating micro-pump, an external tape heater, and a liquid spray tube. The evaporator simulates the output vapor flow from a membrane evaporative chiller in an actual TCS. The vapor production rate is adjusted using the tape heater. A high-accuracy pressure gauge provides precise measurement of the saturation pressure, and consequently, saturation temperature. A T-type thermocouple immersed in the evaporator provides a secondary check of the vapor state leaving the evaporator.

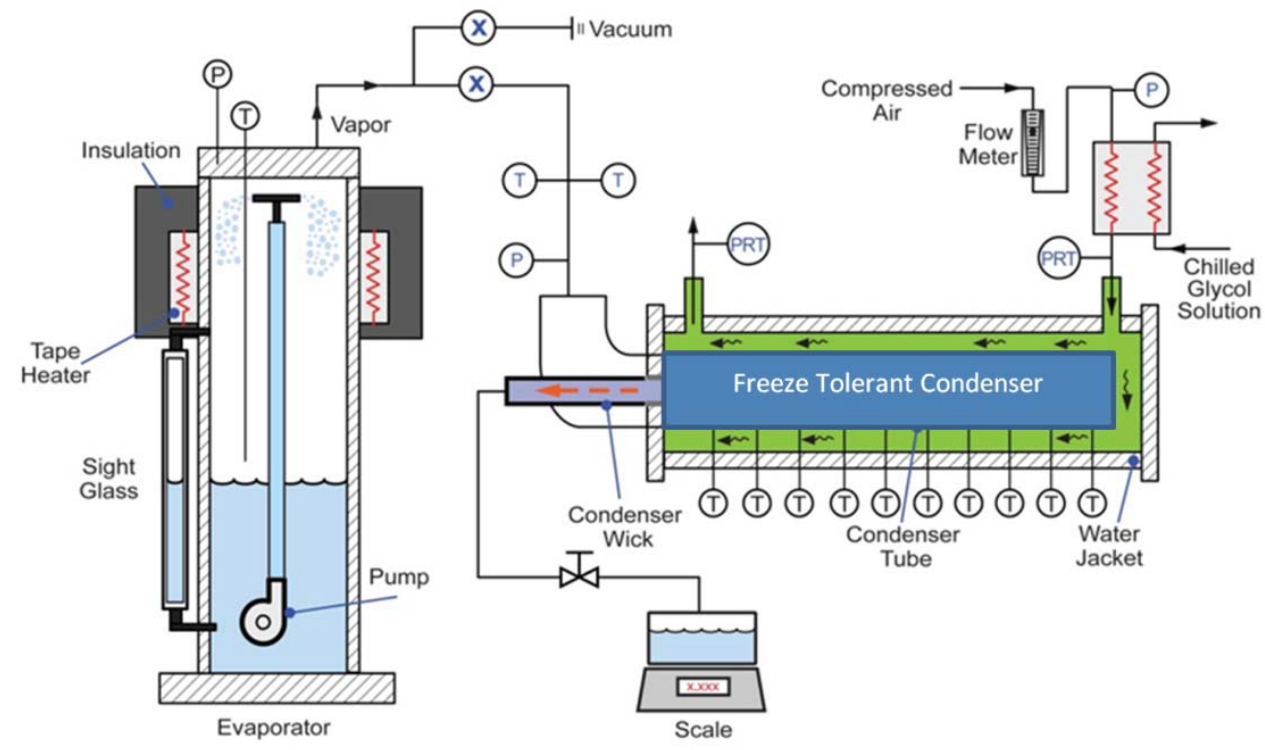

\section{Figure 8. Schematic of Test Facility}

An outer tube surrounding the condenser tube serves as a radiative load simulator. Air circulates through the annular gap between the condenser tube and the simulator shell to convectively cool the condenser. The thermal resistance from the condensed vapor to the air flow is representative of the resistance from the condensing vapor to the radiative heat sink in actual applications. A propylene glycol-water loop flows in a counter-flow heat exchanger to chill the air prior to the radiative load simulator.

Ten thermocouples were installed axially along the condenser tube. The tip of each thermocouple is positioned in the midpoint of the condenser tube wall and reflects the fluid temperature inside the condenser. The array of thermocouples provides information regarding the axial temperature profile inside the condenser, allowing us to observe how the active and frozen condenser lengths change depending on the load. The temperature change of the 
air across the condenser section measured via PRTs coupled with air flow rate measurements provides estimates of the condensing heat load via an energy balance (calorimetry). High accuracy absolute pressure gauges and T-type thermocouples monitor the fluid state at the inlet and outlet of the condenser.

Condenser Performance Testing. Preliminary testing demonstrated three fundamental operating characteristics of the freeze-tolerant radiator technology: (1) self-regulating thermal conductance in response to variable heat loads; (2) effective operation while the condenser is partially frozen; and (3) rapid start-up time from a frozen state.

(1) Self-Regulating Thermal Conductance. Figure 9 provides an example of the consenser's ability to self-regulate its thermal conductance. At $\mathrm{t}=21 \mathrm{~min}$, the condenser vent port is open to a vaccum pump to remove non-condensable gas and the two-phase zone to the far end of the condenser. After closing the vent port, liquid progressively accumulates in the condenser starting at the distal end, illustrating the self-regulating behavior.

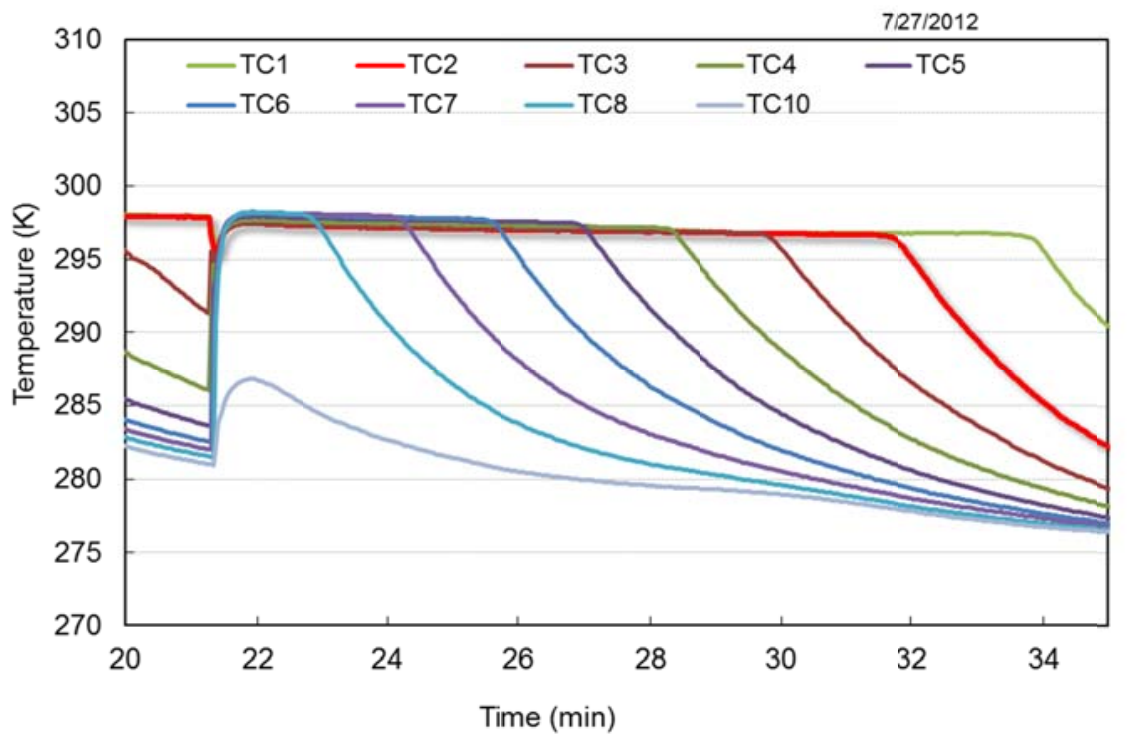

Figure 9. Self-Regulating Response to Evacuation Event

(2) Start-up from Frozen State. The freeze-tolerant radiator is designed to start up quickly from nearly fully frozen states after extended low loading scenarios. Figure 10 shows the thermal response to a uniform increase in the heat sink temperature from a near frozen state. In the initial steady state condition, the completely solid, inactive zone extends up to TC6. TC5 is positioned in the vicinity of the freezing front, while TC4 lies in the middle of the liquid transition zone.

After the heater in the evaporator and cooling air is turned off, residual heat from the evaporator continues to provide vapor to the condenser. The condensation heat conducts axially, pushing the two-phase zone deeper into the condenser while the temperature along the entire transition and inactive regions gradually rises. Once the ice fully melts, the two-phase front quickly penetrates the full length of the condenser. 


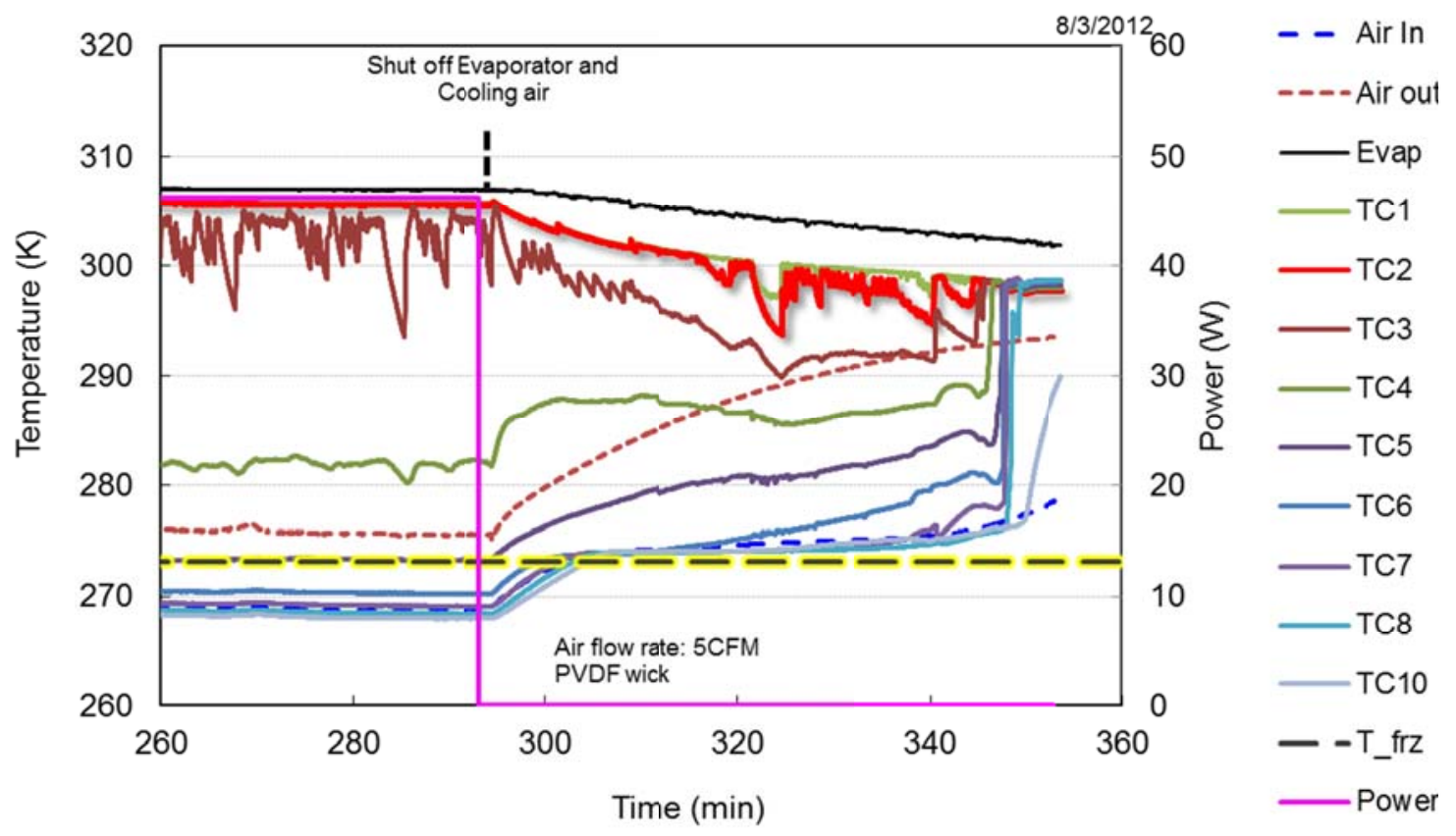

Figure 10. Start-up From Near Frozen State

(3) Freeze-Thaw Cycles. Orbiting spacecraft and periodic equipment operation will subject the radiator to cyclic loading conditions. The radiator must be able to accommodate freezing during cold or low loading phases, and rapidly absorb the heat as heat load increases at other points. The ability of the freeze-tolerant condenser to adjust to such cyclic loading is depicted in Figure 11. In this scenario, the convective cooling air flow rate and inlet temperature remained fixed throughout the test.

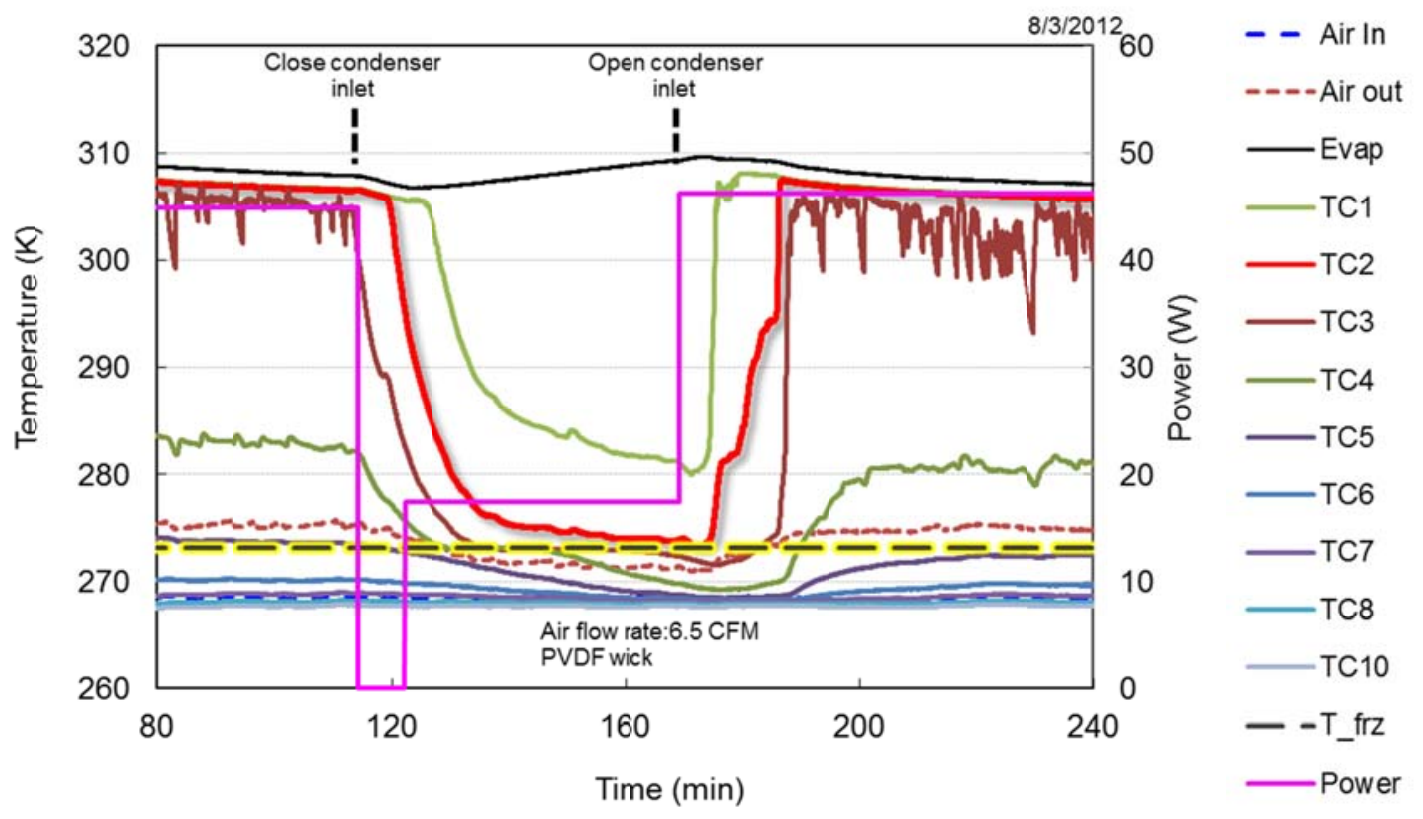

\section{Figure 11. Freeze-Thaw Cycle Behavior}

The condenser starts at a steady condition dissipating $45 \mathrm{~W}$ with an active region extending to TC4. At 115 min, the condenser inlet valve is closed, preventing vapor from flowing into the condenser. At this point, the evaporator heater power is set to $0 \mathrm{~W}$ followed shortly by an increase to $18 \mathrm{~W}$. The condenser increases the inactive region 
from TC5 to TC2. At $170 \mathrm{~min}$, the condenser inlet valve is opened with the heater power returning to $45 \mathrm{~W}$. The active, two-phase zone advances in response to the increased heat load. Axial conduction begins the melting process of the frozen zone. Within 15 minutes, the condenser has regulated itself to a point where it is rejecting the increased load, despite the fact that the melting process is not complete. The latent heat of fusion allows the radiator to accomodate a fast power ramp to its full design load even though the time to reestablish a steady operating state takes nearly 40 minutes. This example clearly demonstrates the freeze-tolerance capability.

\section{Demonstration of Biocide and Corrosion Inhibitors}

Water is the most benign working fluid and has the best transport and thermodynamic properties. However, pure water is corrosive to pipes and heat exchangers made of aluminum alloys. To overcome this problem and to prevent formation of biofilm, corrosion inhibiting and biocidal additives must be introduced in the water. UTAS has identified a promising organic acid-based corrosion inhibition package for water in aluminum heat exchangers. Test results show that the additives are stable over a wide temperature range ( $275 \mathrm{~K}$ to $333 \mathrm{~K}$ ) and through a 24-hour freeze/thaw cycle. Furthermore, short-term corrosion test data indicate greater than an order of magnitude reduction in aluminum corrosion rate. The results of this development effort are briefly discussed below.

An iterative process was undertaken to determine the appropriate concentration of each ingredient in the water-based coolant. This was a balancing act between ideal target concentrations and the solubility of the mixture in the target temperature range ( $275 \mathrm{~K}$ to $333 \mathrm{~K}$ ) and through a freeze/thaw cycle. Solubility was based on visual examination of the solutions to ensure clarity with no signs of precipitation and/or stratification.

CPP (Cyclic Potentiodynamic Polarization) was used as an analysis tool to estimate the corrosion rate of aluminum alloy heat exchanger material in the subject coolant. Tafel extrapolation per ASTM G 102 was performed on the CPP data curves to provide a corrosion rate estimate. This method is also capable of revealing any tendency for pitting for the combination of material and environment (in this case, the subject coolant). Aluminum Alloy 6951 samples were soaked in the coolant solution for approximately 2.5 weeks before testing.

Figure 12 represents the results of a CPP corrosion test. These curves are plots of the log of corrosion current in amperes (amperes per square centimeter for a $1 \mathrm{~cm}^{2}$ sample) as a function of polarization potential in volts vs. a Saturated Calomel Electrode (S.C.E.). The red curves give the raw data, and the green curve shows the curve model used in the Tafel Extrapolation, which gave corrosion rates of 0.229 mpy for this trial.

Figure 13 shows the generic corrosion rate of aluminum as a function of $\mathrm{pH}$ value. At a $\mathrm{pH}$ value of 7.8 (the $\mathrm{pH}$ of the developed coolant), the corrosion rate is $\sim 0.1 \mathrm{~mm}$ per year (or $3.9 \mathrm{mpy}$ ). This was used as a baseline data point to compare to the corrosion rate of $\mathrm{Al} 6951$ alloy coupons submerged in the subject coolant for a two-week period of time. It should be noted that the $\mathrm{pH}$ of water with no buffer is expected to increase over time when in contact with aluminum due to the generation of aluminum hydroxide corrosion products. The corrosion rate, therefore, would be expected to increase over time with no buffer. 


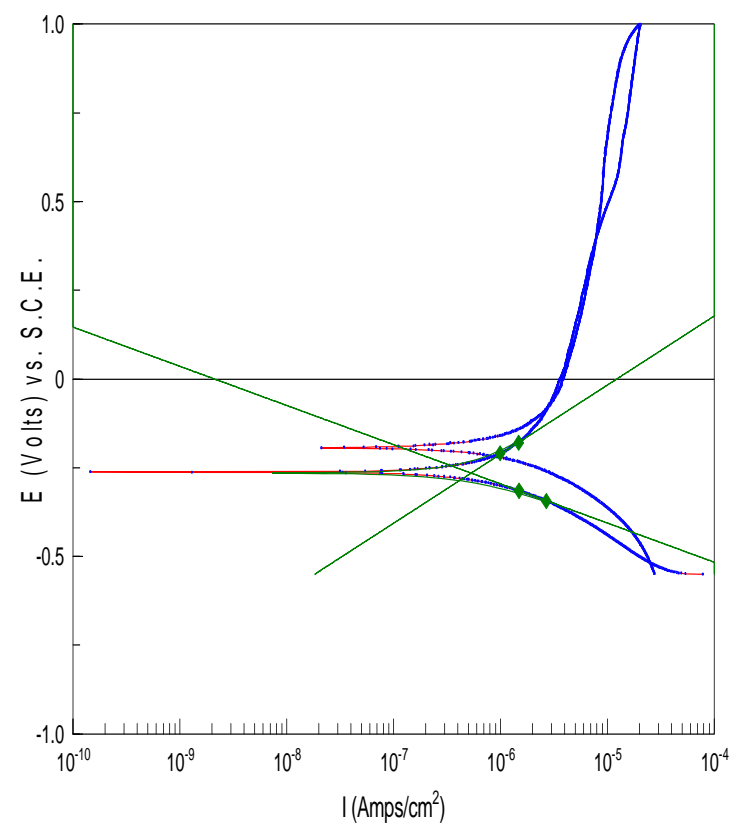

Figure 12. CPP Corrosion Test With Tafel Extrapolation in Water Based Coolant. Corrosion rate $=0.229$ MPY.

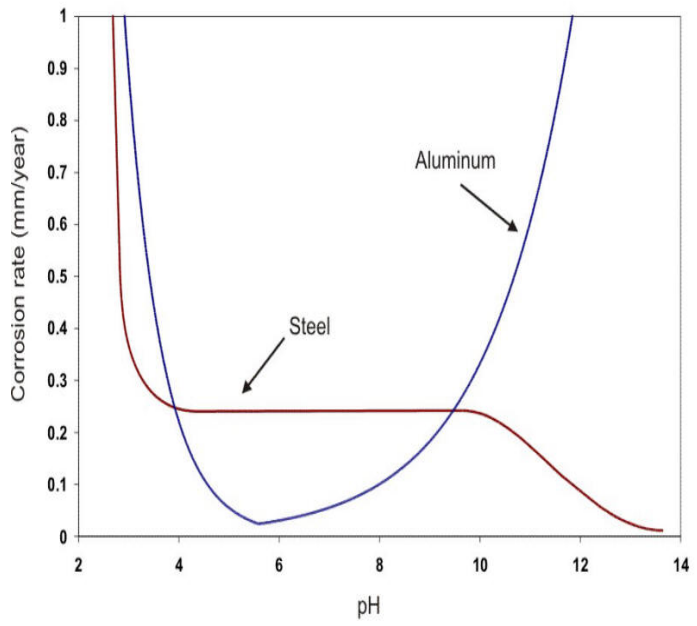

Figure 13. Generic Corrosion Rate of Aluminum in Water $^{1}$

Additionally, UTAS has developed a micro-gravity compatible means to introduce a long-acting, low vapor pressure biocide (ortho-phthalaldehyde) to water-based coolant systems. This biocide has been implemented on the International Space Station in the Internal Active Thermal Control System (IATCS) and has demonstrated high antimicrobial character lasting several months at a time from a single dose.

\section{Performance Comparison With Other Technologies}

A preliminary study was conducted by UTAS to compare the freeze-tolerant radiator and TCS mass to the mass of other TCS and radiator combinations. Comparisons were made to the Orion TCS and radiator and to a lightweight body-panel UTAS radiator design. The preliminary freeze-tolerant radiator design was adjusted to have the same area, thickness, and redundancy as the competing radiators, while the Creare TCS mass and power were adjusted for the specific set of valves and pumps that are unique to that system. As shown in Table 2, the study by UTAS showed that Creare's radiator mass is $43 \mathrm{lb}$ lighter than the lightweight UTAS radiator mass while the system-specific components such as boost pumps, ejectors, and isolation valves only add about $30 \mathrm{lb}$ back to the system. As a result, the total mass and power of Creare's TCS is $13 \mathrm{lb}_{\mathrm{m}}$ less than what might be considered an Orion single-loop TCS. Creare's TCS also benefits from pumping water instead of a 50/50 mix of propylene glycol and water, which saves pumping power in the primary coolant loop. Even when considering the need for boost pumps, the power savings would be about 68 watts compared to the single-loop Orion baseline and 188 watts compared with the two-loop Orion system. More importantly, Creare's TCS has the benefit of using water as a nontoxic coolant inside the cabin, and the coolant is compatible with lightweight aluminum heat exchangers.

\footnotetext{
${ }^{1}<\mathrm{http}: / /$ www.corrosion-doctors.org/Corrosion-by-Water/Constituents.htm>
} 


\begin{tabular}{|c|c|c|c|c|c|c|c|}
\hline \multirow{3}{*}{ Configuration } & \multicolumn{3}{|c|}{ Radiator } & \multirow{3}{*}{$\begin{array}{l}\text { Redundant } \\
\text { Loops? }\end{array}$} & \multirow{3}{*}{$\begin{array}{l}\text { Cabin } \\
\text { Coolant }\end{array}$} & \multirow{2}{*}{$\begin{array}{l}\text { Change in } \\
\text { System } \\
\text { Mass }\end{array}$} & \multirow{2}{*}{$\begin{array}{l}\text { Added } \\
\text { System } \\
\text { Power }\end{array}$} \\
\hline & Area & Thickness & Mass & & & & \\
\hline & $\mathrm{ft}^{2}$ & inches & $\mathrm{lb}$ & & & $\mathrm{lb}$ & $\mathrm{W}$ \\
\hline Orion single-loop system & 249 & 0.040 & 256 & yes & PGW & 0 & 0 \\
\hline Orion two-loop system & 249 & 0.040 & 256 & yes & Water & +300 & +120 \\
\hline $\begin{array}{l}\text { Orion with variable } \\
\text { conductance heat pipes }\end{array}$ & 249 & 0.040 & 256 & yes & Water & +188 & 0 \\
\hline $\begin{array}{l}\text { Orion single-loop } \\
\text { with UTAS radiator }\end{array}$ & 249 & 0.040 & 217 & yes & PGW & -39 & 0 \\
\hline As-designed Creare TCS & 248 & 0.010 & 50.5 & no & Water & -168 & -111 \\
\hline $\begin{array}{l}\text { Adjusted Creare TCS, with } \\
\text { redundant loops }\end{array}$ & 249 & 0.040 & 173 & yes & Water & -53 & -68 \\
\hline
\end{tabular}

\section{Conclusions}

This paper describes early development of a TCS with freeze-tolerant radiators that have self-regulating thermal conductance for future manned spacecraft thermal control. Functional performance testing using convectivelycooled prototypical condenser tubes demonstrated the operating characteristics required for a reliable, freeze-tolerant condensing system, namely (1) self-regulating thermal conductance with short transient responses to varying thermal loads, (2) effective operation in a partially frozen state, (3) fast start-up from fully frozen state, and (4) the ability to survive a fully frozen state without suffering structural damage. A biocide/corrosion inhibitor package was identified to mitigate aluminum corrosion and biofilm fouling, thus enabling water as a coolant for TCS using aluminum heat exchangers. Performance comparison with state-of-the-art designs shows that the TCS with freeze-tolerant radiators offers significant mass and power saving benefits, in addition to having the advantages of minimal moving parts, no active control requirements, high system reliability, and long life.

\section{Acknowledgments}

Work reported here was sponsored by the Crew and Thermal Systems Division at NASA Lyndon B. Johnson Space Center under a Phase I Small Business Innovation Research contract.

\section{References}

Bannon, E. T., Bower, C. E. and Sheth, R. B., "Electrochromic Radiator Coupon Level Testing and Full Scale Thermal Math Modeling for Use on Altair Lunar Lander," International Conference on Environmental Systems, July 2010, Barcelona, Spain.

Bue, G. C. and Makinen, J., "Hollow Fiber Flight Prototype Spacesuit Water Membrane Evaporator Design and Testing”, $41^{\text {st }}$ International Conference on Environmental Systems, July 2011, Portland, Oregon.

Ganapathi, G. B., Sunada, E. T., Birur, G. C. and Miller, J. R., "Design Description and Initial Characterization Testing of an Active Heat Rejection Radiator with Digital Turn-Down Capability,” International Conference On Environmental Systems, July 2009, Savannah, GA.

Nabity, J. A., Mason, G. R., Copeland, R. J. and Trevino, L. A., "A Freezable Heat Exchanger for Space Suit Radiator Systems,” June 2008, San Francisco, CA.

Stephan, R. A., "Overview of the Altair Lunar Lander Thermal Control System Design and the Impacts of Global Access," 41st International Conference on Environmental Systems (ICES 2011), Portland, OR, July 17-21, 2011. 